\title{
La tamsulosina se asocia a eventos adversos oculares severos post-cirugía de catarata
}

Tamsulosin is associated to serious ophthalmic adverse events following cataract surgery

Bell CM y col. JAMA. 2009;301(19):1991-6

\section{Objetivo}

Determinar el riesgo de eventos adversos luego de una cirugía de cataratas en ancianos tratados para la hipertrofia prostática benigna con tamsulosina $u$ otros a-bloqueantes.

\section{Diseño, lugar y pacientes}

Estudio caso-control anidado* de un estudio de cohorte retrospectivo con base poblacional llevado a cabo en Ontario, Canadá. Participaron hombres de 66 años o mayores, operados de catarata entre 2002 y 2007 ( $n=96.128)$. Los datos de los pacientes fueron obtenidos de tres bases de datos sanitarias de Ontario.

\section{Medición de resultados principales}

Solicitud de procedimientos (vitrectomía, aspiración o inyección vítrea, extracción de cristalino dislocado o fragmento cristaliniano, o intercambio de aire o fluido) marcadores de que haya ocurrido desprendimiento de retina, dislocación del cristalino o de un fragmento del mismo o endoftalmitis, dentro de los 14 días posteriores a la cirugía de catarata. El riesgo de estos eventos adversos se comparó entre los hombres tratados con tamsulosina $u$ otros a-bloqueantes y los no expuestos a estas drogas durante el año previo a la cirugía. Se examinaron por separado la exposición reciente (14 días antes de la cirugía) o previa (15-365 días antes de la cirugía). Los casos fueron apareados aleatoriamente a los controles de acuerdo a la edad, el cirujano y el año de la cirugía.

\section{Resultados principales}

Los eventos adversos fueron significativamente más frecuentes entre los pacientes con exposición reciente a tamsulosina pero no a otros a-bloqueantes (ver tabla 1) o con exposición previa a tamsulosina (OR ajustado* 0,94; IC95\% 0,27 a 3,34) u otros a-bloqueantes (OR ajustado 1,08; IC95\% 0,47 a 2,48).

Tabla 1: eventos adversos post-operatorios tras la exposición reciente a a-bloqueantes

\begin{tabular}{l|c|l|c|c} 
Caracteristica & $\begin{array}{c}\text { Gasos } \\
\mathbf{n = 2 8 0}(\%)\end{array}$ & $\begin{array}{c}\text { Controles } \\
\mathbf{n = 1 1 0 2 ( \% )}\end{array}$ & $\begin{array}{c}\text { OR ajustado } \\
\text { (IC95\%) }\end{array}$ & $\begin{array}{c}\text { NND }^{*} \\
\text { (IC95\%) }\end{array}$ \\
\hline No uso & $226(80,7)$ & $950(86,2)$ & 1 (referencia) & - \\
\hline Tamsulosina & $21(7,5)$ & $30(2,7)$ & $2,33(1,22$ a 4,43) & 255 (99 a 1666) \\
\hline Otros $\alpha$-bloqueantes & $21(7,5)$ & $88(8,0)$ & $0,91(0,54$ a 1,54) & - \\
\hline
\end{tabular}

\#Número necesario para dañar (NND*) para un riesgo de base de eventos de $0,3 \%$.

\section{Conclusiones}

La exposición a tamsulosina durante de los 14 días previos a la cirugía de catarata se asoció significativamente a eventos adversos oculares severos postoperatorios. No se encontraron asociaciones significativas con la exposición a otros a-bloqueantes.

Palabras claves: Tamsulosina, cirugía de catarata, eventos adversos postoperatorios, hiperplasia prostática.

Key words: Tamsulosin, Cataract Surgery, Postoperative Adverse Events, Prostatic Hyperplasia.

Fuente de financiamiento: Canadian Insitutes of Health Research; Institute of Nutrition, Metabolism, and Diabetes, y Ontario Ministry of Health and Long-Term Care.

\section{Comentario}

La hiperplasia prostática benigna (HPB) afecta aproximadamente al $50 \%$ de los hombres mayores de 50 años y al $90 \%$ de los mayores de $85^{1}$. La cirugía de catarata se encuentra entre los procedimientos quirúrgicos más frecuentemente realizados en los países desarrollados ${ }^{2}$. Por otro lado, se espera que la incidencia de ambos eventos (HPB y catarata) aumente a medida que la población envejezca. El antagonista a1-adrenérgico tamsulosina es uno de los medicamentos más frecuentemente indicados para el tratamiento de la HPB. Esta droga relaja el músculo liso prostático y del cuello vesical, mejorando así el vaciamiento de la vejiga y disminuyendo la frecuencia miccional. Estudios en animales e in vitro han mostrado que la tamsulosina tiene especial afinidad por los receptores del subtipo a1A, que constituyen el $70 \%$ de los receptores a1 de la próstata humana. Se cree que este subtipo prevalece en el músculo liso dilatador del iris. Chang y Campbell postularon que la tamsulosina bloquea la contracción del músculo dilatador del iris, y este tono muscular alterado causaría lo que estos autores describieron en 2005, como el Síndrome del Iris Fláccido Intraoperatorio (IFIS) ${ }^{3,4}$. Además de un conjunto de alteraciones características durante la cirugía de catarata, relacionadas con la flaccidez del iris, la miosis progresiva y la tendencia del iris a prolapsar por la herida (con mayor índice de complicaciones quirúrgicas indeseables) los autores reportaron una fuerte asociación entre la aparición de este síndrome y la tamsulosina. El presente estudio suma a las complicaciones intraoperatorias del IFIS ya descriptas en numerosos trabajos, el aumento de eventos adversos oculares severos postoperatorios en pacientes con exposición reciente a tamsulosina.

\section{Conclusiones del comentador}

Si bien no se ha demostrado aún la utilidad de la interrupción de la droga antes de la cirugía, identificar su uso en el paciente que será intervenido, con la consiguiente advertencia al cirujano, le permitirán a éste tomar los recaudos y realizar las maniobras intraoperatorias necesarias para prevenir complicaciones. La indicación de otro tipo de droga en pacientes que se operarán de catarata en el corto plazo es también otra opción a considerar.

Ana Gabriela Palis [ Servicio de Oftalmología, Hospital Italiano de Buenos Aires. gabriela.palis@ hospitalitaliano.org.ar ]

Ver glosario*

Recibido el 10/12/09 y aceptado el 25/01/10.

Palis A. La tamsulosina se asocia a eventos adversos oculares severos post-cirugía de catarata. Evid Act Pract Ambul. 13(1) 16. Ene-Mar 2010. Comentado de: Bell $\mathbf{C}$ y col. Association between tamsulosin and serious ophthalmic adverse events in older men following cataract surgery. JAMA. 2009;301(19):1991-6. PMID:19454637.

\section{Referencias}

1. Ziada A y col. Benign prostatic hyperplasia: an overview. Urology 1999;53:1-6.

2. American Academy of Ophthalmology. Cataract in the Adult Eye, Preferred Practice Pattern. San Francisco: American 2.Academy of Ophthalmology, 2006. Available at: www.aao.org/ppp.

3. Chang D y col. Intraoperative floppy-iris syndrome associated with tamsulosin. J Cataract Refract Surg 2005;31:664-73.

4. Chang D y col. Prospective multicenter evaluation of cataract surgery in patients taking tamsulosin (Flomax). Ophthalmology 2007;114:957-64. 